\section{Evolución de la endemia de la lepra en Brasil}

\section{Time Trends of Hansen's Disease in Brasil}

\section{Maria da Conceição Cavalcanti Magalhães ${ }^{1}$ \\ Luisa Iñiguez Rojas ${ }^{2}$}

'Programa Nacional de Eliminação da Hanseníase, Secretaria de Vigilância à Saúde (SVS) - Ministério da Saúde (MS)

${ }^{2}$ Centro de Estudios de Salud y Bienestar Humano.

Universidad de La Habana, Habana, Cuba

\section{Resumo}

La presente investigación se propone abordar la distribución territorial de la lepra en Brasil con el objetivo de identificar las regularidades de su diferenciación espacial y aportar conocimientos que permitan progresar en la comprensión de los procesos de su producción social. Fueron calculadas las tasas anuales de detección de casos, brutos y específicos, para las regiones y estados del país. La evolución espacio-temporal fue realizada mediante la construcción de series históricas. Se expone como substrato de los análisis una síntese sobre la distribución histórico-geográfica de la endemia en Brasil.

La serie histórica del período 1960 2002, permite comparar las tasas de detección de las regiones, en la primera década, con las tasas más bajas, a excepción de la región Norte, que se destaca com la mayor detección. Todas las regiones elevan la detección a partir de la década del 70, siendo la Sul la de detección más baja. Las regiones Norte y Centro Oeste, mantienen las más altas detecciones de casos y los mayores incrementos, lo cual evidencia la desigual evolución regional de la endemia en el país.

La permanencia histórica de elevada incidencia de la lepra en la región Norte, en el estado de Maranhão y Pernambuco en la región Nordeste, del estado de Paraná en la región Sul, así como relativamente bajas incidencias en el resto de esta última región, sugieren la existencia de contextos geográficos de diferente vulnerabilidad a la producción social de la lepra, y de nuevos componentes en la organización espacial en algunos territorios.

Palavras-chave: Lepra. Tasa de detección. Séries históricas. Diferenciación espacial. Producción social. 
The present investigation intends to study the territorial distribution of leprosy in Brazil in order to identify the regularities of its differentiation, and to contribute to the knowledge that allows progress in the understanding of the processes of its social production. Time trends of global and specific leprosy detection rates were calculated for regions and states of the country. A synthesis of the historicalgeographical distribution of the endemic in Brazil is shown as substratum of the analysis.

The historical series of the 1960-2002 period allows the comparison of detection rates for regions, in which the first decade presents the lowest detection rates, except for the North region that stands out with the highest detection rate. Detection grew for all regions as of the 1970's, with the lowest detection in the South region. The North and Center West regions, maintained the highest detections of cases and the largest increments, which is an evidence of the unequal regional development of the endemic in the country.

The historical stability in the high incidence of leprosy in the entire North region, in the states of Maranhão and Pernambuco in the Northeast region, and in the state of Parana in the South region, as well as the low incidence of leprosy in the other two states of the South region suggests the existence of geographical contexts with different vulnerability to the social production of leprosy, and new components in the organization of space in some territories.

Keywords: Leprosy. Detection rate. Time trends. Space differentiation. Social production

\section{Introducción}

La presente investigación se propone abordar la distribución territorial de la lepra en Brasil con el objetivo de identificar las regularidades de su diferenciación espacial y aportar conocimientos que permitan progresar en la comprensión de los procesos de producción social de la entidad, visto o Brasil tener en las últimas décadas la situación más desfavorable de la región de las Américas. La lepra en Brasil es por tanto considerada un problema de salud pública y su programa de eliminación está entre las acciones priorizadas por el Ministerio de Salud. La reducción de la prevalencia observada a partir de 1991, no coincide con la disminución en la detección de casos ${ }^{1}$.

Los avances en el conocimiento científico de las causas de la enfermedad, del desarrollo terapéutico, apoyado en las últimas décadas en la biotecnología, ingeniería genética, biología molecular, han logrado que en la actualidad, se acepte que la enfermedad tiene cura, y que pueden ser evitados deformaciones y sufrimientos a ella asociados. Mientras permanecen los estudios sobre la posible inmunización por vacuna específica.

La Organización Mundial de la Salud propuso en la $44^{\text {a }}$ Asamblea Mundial de Salud "eliminar la lepra como un problema de salud pública" en el 2000 esto es, reducir la prevalencia a menos de un caso por diez mil habitantes. Para ello los países endémicos proyectaron expandir las actividades de búsqueda de casos y ofrecer los esquemas de la polioquimioterapia (PQT) a todos los pacientes.

Varias de las principales áreas históricamente endémicas en el mundo se encuentran en climas tropicales, con elevada temperatura y precipitaciones, mientras en otras de climas templados y fríos, la lepra tuvo elevadas incidencias, eliminándose posteriormente sin una definitiva explicación. En la actualidad no quedan dudas de su distribución agrupada en zonas tropicales. 
Por otra parte es en general aceptada su asociación con desfavorables dimensiones de las condiciones de vida como la económica, higiénico-sanitaria, y biológica. La relación entre pobreza y lepra, ratifica el papel del deterioro social, en su producción, no obstante en algunos países considerados hiperendémicos, estudios detallados en áreas muy pobres han hallado muy bajas prevalencias, lo cual sugiere la existencia de condiciones que no propiciaron la entrada o sobrevivencia del agente patógeno.

La presentación geográfica en focos, la mayoría de los cuales no tienen una explicación definitiva, la prolongada evolución subclínica de la enfermedad, los intensos movimientos migratorios y desplazamientos de población en las últimas décadas, y en especial la concentración de población en ciudades, hacen de la geografía de la lepra uno de los más difíciles capítulos de la nosogeografía mundial, regional y local.

La investigación se desarrolla a partir de los casos detectados. Fueron calculadas las tasas anuales de detección de casos, brutos y específicos, según grupo de edades, sexo, forma clínica y grado de incapacidad física en el momento del diagnóstico, para las regiones y estados del país. La evolución espacio-temporal fue realizada mediante el cálculo de las tasas anuales de detección y la construcción de series históricas. Para el procesamiento de los datos fueron utilizados: el TABWIN y el SPSS 10.0. Se expone como substrato de los análisis una síntese sobre la distribución histórico-geográfica de la endemia en Brasil.

Fueron fuentes esenciales el SINAN (Sistema de Información de Agravos de Notificación) y los informes anuales de evaluación del Àrea Técnica de Dermatologia Sanitária, así como informaciones y tablas elaboradas por el IBGE (Instituto Brasileiro de Geografia y Estadística).

\section{Apuntes históricos sobre la endemia de la lepra en Brasil}

Los primeros casos de lepra en Brasil fueron registrados en 1600, en Rio de
Janeiro, donde más tarde sería creado el primer lazareto. Otros casos se identificaron en Bahía, Pernambuco y Pará. Esto llevó a las autoridades, a pedir a Portugal la toma de medidas, que en realidad fueron implantadas dos siglos después, con el reglamento de combate a la enfermedad por la orden D. João V.

Según Belmiro Valverde ${ }^{2}$, la diseminación de la lepra por el país, acompañó los flujos migratorios, responsables por su distribución desde el periodo colonial, a partir de los casos de Rio de Janeiro, Bahía y Recife, ciudades éstas que tienen en común el hecho de haber sido los principales puertos de entrada de europeos y africanos. Por otra parte, el propio autor refiere que en las provincias de São Paulo, Rio de Janeiro, Minas Gerais, Bahía, Pernambuco y Pará donde la agricultura fue más desarrollada, y se encontraban en mayor número los esclavos, fueron las primeras donde se instaló la endemia, y ocurrió a partir de ellas la más intensa diseminació ${ }^{2}$.

En el año 1890 se registraban en Brasil 1640 enfermos, 220 en Pará y Maranhão, 580 en Minas Gerais, 480 en São Paulo y 360 en el resto del país ${ }^{3}$. El incremento de casos en años posteriores, sugieren la progresión de la endemia desde finales del siglo XIX.

Llama la atención que datos estadísticos de fechas similares al presentado, refieran en conjunto los casos de Pará/ Amazonas/Maranhão, como si se aceptase la proximidad en la evolución de la endemia en estos tres estados. En 1890 estos estados concentraban poco más del $6 \%$ de la población y el $14 \%$ de los casos, mientras en 1926 la población de estos estados representaba alrededor del $1 \%$ del país y concentraban el $13 \%$ de los casos totales, lo cual ratifica este territorio como históricamente leprógeno.

\section{Distribución histórico-geográfica de la endemia en el país}

Hay una amplia documentación histórica de la lepra en el país, aunque está fundamentalmente referida a los leproserias y 
otras instalaciones similares de reclusión y atención a los enfermos. La minuciosa búsqueda de documentos asociados a la enfermedad en la obra monumental "História da lepra no Brasil" compilada por Heraclídes Cesar de Souza Araujo publicada en 1956, permite también la reconstrucción aproximada de la evolución espacial y la diferenciación territorial de la endemia en el país, desde finales de siglo XIX e inicios del XX33.

A continuación se exponen los principales argumentos encontrados en esta obra sobre la evolución histórica territorial de la endemia.

Según Souza Araujo en 1889 Alfredo da Matta consideró la enfermedad rara y de baja prevalencia en Amazonas4. Llama la atención que en el conocido informe sobre las condiciones higiénicas de la Amazonia, coordinado por el sanitarista Oswaldo Cruz, otro sanitarista, Carlos Chagas plantea, pocos años después la gravedad de este flagelo en el norte del país, si comparado con las regiones del sul del país, llamando la atención a su presentación tanto en las regiones más pobladas, como en las interiores.

La lepra no solo en Amazonas, sino en todo el norte de Brasil era considerada una calamidad social a inicios del pasado siglo $^{4}$. En esta epoca en el estado de Pará fue obervada la mayor concentración de casos en Cameta y Santarén, y su extinción en Gurupa ${ }^{4}$.

En relación con la región nordeste varios documentos citados por Souza, permiten reconocer las diferencias interestatales. En trabajos de 1912 y 1918, Arthur Neiva y Belisario Penna, consideran que la lepra en Piauí y Ceará era una enfermedad rara, e ignorada. Según Belisario Penna en Rio Grande del Norte y Paraiba era desconocida hasta 1920, cuando las campañas de saneamiento rural comienzan a desarrollarse.

Carlos da Costa Ribeiro en 1921, observa que la lepra se concentra en Ceará en la parte más próxima al litoral, que durante la estación lluviosa queda casi sumergida. El alto sertão y la mitad sul del estado no presentan casos. Ribeiro llama la atención sobre el hecho de que el regreso de personas enfermas de lepra, que trabajaban en la explotación del caucho en el interior de Amazonas y se vinculaban al "boom" de las plantaciones de algodón, diseminaban la lepra en algunos casos y en otros casos no. Considera Ipú como un foco activo caracterizado por el gran comercio con el Amazonas ${ }^{4}$.

Una interesante observación que reitera la baja incidencia de la entidad en Rio Grande del Norte, es la de Magalhães en 1900 , donde refiere que la entidad no encontró terreno favorable para su evolución ${ }^{5}$. Se considera rara pero más frecuente en el litoral que en el sertão ${ }^{3}$.

En Sergipe, José Rodríguez da Costa publica en 1918, un artículo sobre la frecuencia de la lepra, donde la considera rara, en comparación con otros estados. La parte norte del estado es inmune, y las localidades vecinas del Rio San Francisco, presenta baja frecuencia de casos. En el estado de Bahía, por el contrario Nina Rodríguez, plantea que la lepra esta diseminada y solo se salvan una parte de sus vastos sertões 4

Marcelino Rodríguez expuso el trabajo "Frecuencia de la lepra en el Estado de Maranhão" en el VII Congreso Brasileño de Medicina celebrado en Rio de Janeiro en 1918, donde observó que la lepra se distribuye en la planicie maranhense, entre los rios Itapecurú y Turyassú, y destaca que la mitad sul del estado formado por el Alto Sertão, no presenta casos $^{3}$.

En el territorio que en la actualidad ocupa la región centro oeste del país, hemos encontrado pocas alusiones a la evolución espacial de la lepra en la obra de Souza-Araujo. En Mato Grosso se considera la existencia de un "silencio" informativo. Es de interés la observación de que en la localidad Caldas da Rainha de aguas termales, se envían a curarse enfermos de Para, previo acuerdo de los gobernadores respectivos. Se considera que en el sul del estado de Goiáis hay muchos casos y en la parte norte no hay casos.

En el estado de Minas Gerais, se cita 
que la lepra no tiene la gran diseminación de otras estados del país y en Belo Horizonte no es una enfermedad de niños, con predominio de la forma tuberculoide ${ }^{4}$.

Importantes observaciones sobre la geografía de la lepra son hechas por Fernando Terra, para el estado de Rio de Janeiro, en trabajo presentado al Primer Congreso Sulamericano de Dermatología y Syphiligraphia”. Se considera su presentación en focos en los suburbios, barrios periféricos a la ciudad. Por otra parte, se plantea que la región de Saquarema concentra muchos enfermos, y se bosqueja probablemente una de las primeras referencias a la participación de factores del ambiente físico en su difusión, al citarse su asociación a los lagos de esta región ${ }^{4}$.

Según trabajos de Emilio Ribas sobre la frecuencia de lepra en São Paulo, en 1918, de los 171 municipios del estado, 126 tenían enfermos y se coloca la posible participación de mosquitos en la transmisión de la enfermedad ${ }^{4}$.También en el trabajo Geografía Médica en el Estado de São Paulo presentado por Seabra y Silveira en el Primer Congreso Médico Paulista, en 1916, se recoge la amplia distribución de casos en el estado, tanto en la capital, como en las ciudades y poblados del interior. Se define como el área de más casos, la comprendida entre São Paulo y Paraná ${ }^{4}$.

En la región sul del país, existe una amplia documentación sobre la lepra y se considera rara en los tres estados que la integran. En el estado de Rio Grande del Sul es más frecuente en el litoral que en el interior ${ }^{4}$.

Una particular precisión sobre la frecuencia y distribución de la lepra en el estado de Paraná fue realizada en 1921. Los primeros casos del estado de Paraná, se consideran importados de São Paulo y Minas Gerais y se calcula la existencia de entre 600 y 800 enfermos. Se observa la mayor frecuencia en el límite con el estado de São Paulo y de europeos en el registro de casos, que se considera llegaron enfermos ${ }^{4}$. Llama la atención el funcionamiento de un consorcio para el control de focos, integrado por siete municipios: Castro, Jaguarialva, Sao José de Boa Vista, Tibagy, Rio Negro, Thomazina, y Guarapuava donde se reportan más de 200 enfermos.

Analizando el contexto histórico de ocupación espacial de la región nordeste, se observa que los procesos de colonización de la franja litoral atlántica, se centraron en la producción azucarera, con el fomento de plantaciones cañeras que avanzaron rápidamente sobre los bosques (Mata Atlántica). Fue una característica del poblamiento la llegada de importantes contingentes de población africana, como mano de obra esclava, que trabajaban y sobrevivían en trágicas condiciones de vida. Los centros principales de ocupación, de mejores condiciones productiva, se concentraron en los estados de Rio de Janeiro, Bahía y Pernambuco. En los dos últimos estados se reconocen los flujos y reflujos cíclicos de la población de los sertões al litoral, a expensas de las grandes secas ${ }^{6}$.

Mientras, en la región sul del país, fue fomentada la colonización de familias, sistema conocido como "colonización de parejas”, asentados en determinadas parcelas para desarrollar la agricultura, la gran mayoría colonos provenientes de las islas Azores. Fue este tipo de inmigración el sustrato principal del poblamiento de Santa Catarina y Rio Grande del Sul ${ }^{6}$.

A inicios del Siglo XIX, se considera que los principales núcleos de desarrollo económico presente y pasado del país, se localizaban en Maranhão a expensas del desarrollo de las plantaciones de algodón, en el extremo nordeste, el Recóncavo Baiano y Rio de Janeiro por la explotación azucarera y en áreas de explotación minera de Mato Grosso, Goiás y Minas Gerais ${ }^{6}$. A finales de siglo, el "boom" de la explotación del caucho, en el interior de la Amazonia, abriría frentes importantes de desarrollo económico en Amazonas y Pará, que promueven el crecimiento de la población en especial por inmigrantes. São Paulo desarrollado en especial por la economía cafe- 
talera y Rio de Janeiro donde se asentara la metrópoli, eran regiones de intensa concentración de la población.

Una especial consideración sobre la geografía de la lepra en Brasil, es aquella realizada por Belisario Penna en 1926, quien considera la existencia de dos grandes areales de lepra en el país. Uno en la parte norte, integrado por los estados de Amazonas, Pará y Maranhão, y otro al sul integrado por los Estados de São Paulo y Minas Gerais. En ellos se concentraban el $80 \%$ de los enfermos del país, con aproximadamente el $40 \%$ de la población.

Según datos de la inspectora de salud para el 31 de julio de 1927, la distribuición de casos por estados era la siguinte (Tabla 1).

De esta forma, al margen de las diferencias en la confiabilidad de los registros, o la capacidad diagnostica de los estados, las anteriores informaciones coinciden aproximadamente con las observaciones realizadas por Belisario Penna, en 1926.

Según la información histórica analizada podemos considerar la existencia de dos epicentros en la expansión de la endemia en el país. Uno localizado en la Amazonía y otro en las áreas y ciudades de la región sudeste de mayor poblamiento, que comprenden 5 de los 21 territorios existentes a finales de la década del 20.
Ambos espacios concentraban el $80 \%$ de los casos conocidos.

Es esta una observación geográfica que si bien sugieren contextos similares y desfavorables de reproducción social, parecen obedecer a procesos diferentes de ocupación del espacio, y de condiciones físico ambientales, que pueden por otra parte relacionarse con la distribución geográfica del agente patógeno.

\section{La endemia en Brasil en el año de 2002}

La tasa de detección de casos de lepra, en Brasil en el año 2002, fue de 2,69 por 10.000 hab, con 47026 casos nuevos detectados. La región Norte presentó la mayor tasa del país, con 7,73 por 10000 hab., seguida de la región Centro-Oeste con 6,61 por 10000 hab., considerándose, según parámetros aceptados por el Ministerio de la Salud como áreas hiperendémicas. La región nordeste con una tasa de 3,23 por 10000 hab. y el sudeste con 1,46 por 10000 hab. fueron consideradas de alto riesgo. Mientras, la región sul con una tasa de detección de 0,75 por 10000 hab. se evaluó como de media intensidad de transmisión.

La Amazonia Brasilera contenida en la región norte, a pesar de concentrar apenas el $10,4 \%$ de la población del país, contiene el $44,1 \%$ de la detección, con una tasa

Tabla 1- Tasas de prevalencia y casos de lepra por estados - 1927

Table 1 - Prevalence rates and number of cases of Leprosy in Brazilian States - 1927

\begin{tabular}{lrrlrr}
\hline Estados & Tasas* $^{*}$ & Casos & Estados & Tasas & Casos \\
\hline Amazonas & 20,8 & 828 & Pará & 21,1 & 2540 \\
Maranhão & 6,8 & 680 & Piauí & 0,6 & 46 \\
Ceará & 3,1 & 457 & RG del Norte & 2,4 & 89 \\
Paraíba & 0,3 & 29 & Pernambuco & 5,6 & 355 \\
Alagoas & 0,3 & 32 & Sergipe & 0,2 & 9 \\
Bahia & 0,2 & 82 & Espírito Santo & 0,4 & 22 \\
Río de Janeíro & 0,5 & 84 & DistritoFederal** & 11,5 & 167 \\
Minas Gerais & 0,9 & 601 & São Paulo & 8,4 & 4620 \\
Paraná & 4,6 & 380 & Santa Catarina & 1,3 & 106 \\
RG do Sul & 0,2 & 64 & Mato Grosso & 3,3 & 97 \\
Goiás & 0,1 & 2 & Total & $\mathbf{3 . 6}$ & $\mathbf{1 2 8 3 0}$ \\
\hline
\end{tabular}

*por 10.000 hab./ * per 10,000 inhab.

**Distrito Federal era en esta época la ciudad de Rio de Janeiro y su área de influencia. Fuente: Souza Araujo 1956, IBGE.

** The Federal District at this time was the city of Rio de Janeiro and its area of influence. Source Souza Araujo 1956, IBGE 
de 12,63 por 10000 hab., en cuanto la tasa del resto del país es de 1,85 por 10000 hab., lo cual evidencia la focalización territorial, incluso a este nivel de observación.

El estado de Mato Grosso (12,02 por 10 000 hab.) obtiene la tasa más alta de detección entre el total de los estados de la Federación, seguido de Pará $(9,25$ por 10 000 hab.), Tocantins (9,17 por 10000 hab.), Roraima (8,50 por 10000 hab.), Rondônia (8,18 por 10000 hab.) y Maranhão (8,07 por 10000 hab.). Son también elevadas, por encima de la media nacional, las tasas de los estados de Goiás (7,41 por 10000 hab.), Piauí (6,37 por 10000 hab.), Espírito Santo (5,33 por 10000 hab.), Pernambuco (3,67 por 10000 hab.) y Mato Grosso do Sul (3,02 por 10000 hab.).

Brasil, presentó en el año 2002 una tasa de prevalencia de 4.42 por 10000 hab., com 77154 casos registrados. Por macro regiones se observan las tasas más elevadas en el en el Centro- Oeste (11.77), Norte (8.73) y Nordeste $(6,04)$, que de forma similar a la detección, las sitúa muy encima de la media nacional. Las regiones Sudeste (2.41) y Sul (1.43) se consideran de media prevalencia.. Esta tasa según estados arroja que Mato Grosso (24.81), Piauí (16.63), Roraima (14.53), Goiás (12.65), Pará (9.91), Rondônia (9.41), Tocantins (8.67) y Pernambuco (8.51), presentan la situación más desfavorable del país.

Si se utiliza la razón de prevalencia/ detección, observamos que para Brasil este indicador es de 1,64, y varía de 1.12 en el norte, a 1.91 en el sul, de forma que en la región norte puede considerarse que la prevalencia conocida es más próxima a la real. El resto de las regiones presentan los siguientes valores: 1.86 para el Nordeste, 1.78 para o Centro-Oeste, 1.65 para el Sudeste.

Entretanto cuando se analiza esta razón por estados vemos que Piauí, Ceará, Rio Grande do Norte, Paraíba, Pernambuco, Bahia, y Paraná presenta valores por encima de 2.0, lo cual pudiera explicarse por deficiencias operacionales, en el acompañamiento de los casos o del Siste- ma de Información. Por el contrario, los estados de Rondônia, Amazonas, Alagoas y Espírito Santo, obtienen una razón cerca de 1.0, lo cual correspondería a mejores condiciones operacionales y seria lo esperado con los esquemas de PQT de corta duración.

La evolución de la detección según edad, sexo, formas clínicas y grado de incapacidad física, en el momento del diagnóstico

La tasa de detección de casos nuevos en menores de 15 años, para el año 2002, en Brasil fue de 0.70 por 10000 hab., presentando por regiones, amplias variaciones que van de 2.23 por 10000 hab. en la Norte, 1.24 por 10000 hab. en la CentroOeste, y 0.87 por 10000 en la región Nordeste, todas por encima de la media nacional, y de 0.30 por 10000 hab. y 0.07 por 10000 hab., en las regiones Sudeste y Sul respectivamente.

La tasa de detección de casos nuevos en menores de 15 años, está relacionada con el nivel de endemicidad, y refleja la exposición precoz al M. Leprae . Para el periodo de 1985 a 2002, hubo un incremento en Brasil del $16,6 \%$, con variaciones extremas en la regiones, de $335,0 \%$ en la Nordeste, a negativos de -30 en la región Sul, y - 12 en la Norte. En las regiones CentroOeste y Sudeste los incrementos de la detección en este grupo de edades fueron elevados con valores de 53,1 y 42,9\%, respectivamente.

Rondônia, Acre, Amazonas y Amapa, son responsables por el decrecimiento de la región Norte, en cuanto Roraima, Para y Tocantins presentan crecimientos positivos. En la región Nordeste hubo incrementos en todos lo estados con variaciones de $420,7 \%$ en Piauí a $24,1 \%$ en Alagoas. Notables incrementos en las notificaciones de menores de 15 años se observan en Maranhão con 246,7, Paraiba 238,9\%, 183,3 \% en Ceará, $157,1 \%$ en Rio Grande del Norte, $100 \%$ en Bahía, $72,7 \%$ en Sergipe y 57,9\% en Pernambuco. 
En la región Centro-Oeste, el estado de Mato Grosso sobresale con un incremento de $431,0 \%$, en la detección de casos nuevos en menores de 15 años, mientras el resto de los estados presentan crecimiento de $69,4 \%$ como Mato Grosso do Sul o decrecimientos como en el Distrito Federal con $-54.4 \%$ y Goiás con $-18,2 \%$. En el sudeste, Espírito Santo es el estado de mayores incrementos con $365,5 \%$, seguido de Rio de Janeiro con 203,0\%, mientras São Paulo decrece en $-25,0 \%$.

En relación a la distribución de casos por sexo, en Brasil, en el periodo se observa una tasa para el sexo masculino de 3.1/ 10.000 y de 2.5/10.000 para el femenino. Tasas más elevadas para el sexo masculino, se observan en todos los estados de las regiones Norte y Centro-Oeste, en cuanto en el resto de las regiones las tasas no presentan diferencias por sexos, a excepción de Maranhão con una tasa más elevada del sexo masculino.

La tasa de detección de casos nuevos por forma clínica, también está asociada al nivel de endemicidad ${ }^{8}$, de forma que la detección de formas tuberculoides (T), sería un indicador de la tendencia de la endemia. Según forma clínica $(\mathrm{T})$ para el período 1985-2002, se observa la caída porcentual de los estados de más bajas tasas de detección y el incremento de los percentajes de esta forma en los casos nuevos de los estados de más altas tasas de deteccion.

Los estados de Rio Grande do Sul, Santa Catarina, Minas Gerais y Goiás presentan los más altos porcentajes de formas multibacilares, en cuanto Piauí, Sergipe, Alagoas, Rondônia, Tocantins, Espírito Santo y Mato Grosso los más bajos. Ello indica una cierta coherencia entre la tendencia de crecimiento observada, e indica que se enferman personas con mayor inmunidad, lo cual sugiere una elevada concentración de bacilos circulantes.

Por formas clínicas en el momento de la detección, se observa una diferencia de sexo. La razón hombre-mujer es de 0.75:1 en la forma indeterminada, de 0.78:1 en la forma tuberculoide, de 1.46:1 en la dimorfa y de 2.36:1 en la virchowiana. Si realizamos este análisis para las formas multi y paucibacilares observamos que esta razón es de 1.75:1 para las formas multibacilares y de 0.78:1 para las formas paucibacilares.

Para analizar si el diagnóstico está ocurriendo precoz o tardíamente se utiliza el porcentaje de grado de incapacidad entre los casos nuevos evaluados. En el año 2002, fueron evaluados el $69,0 \%$ de los casos nuevos y de estos el 7,9\% presentaban incapacidades físicas, esto es, disminución de sensibilidad protectora en los ojos, manos o pies y $4 \%$ ya presentaban deformidades.

Presentaron los mayores porcentuales de casos nuevos con deformidades: Rio Grande do Sul $(13,46 \%)$, Minas Gerais $(9,49 \%)$, Roraima $(8,71 \%)$, São Paulo $(8,04 \%)$, Santa Catarina $(7,69 \%)$, Paraná (7,52\%), Distrito Federal (7,07\%) y Rio de Janeiro (6,93\%), lo cual puede significar un diagnóstico tardío de la enfermedad.

El modo de detección de casos puede ser utilizado como indicador del conocimiento y la motivación de la población por el diagnóstico. Entre los casos nuevos del año 2002, aproximadamente el $40 \%$ fue detectado por demanda espontánea, seguido del 33,6\% encaminado por los servicios de atención primaria. Apenas el 6,3\% de los casos fueron descubiertos mediante el examen de comunicantes. Este bajo porcentaje puede ser explicado por el número de contactos examinados que es cuasi siempre menor del 30\% de los comunicantes registrados ${ }^{9}$.

\section{Diferenciación territorial de la endemia desde la segunda mitad del siglo XX. Regiones y Estados}

La serie histórica del período 1960 2002, permite apreciar la proximidad de las tasas de las regiones, en la primera década, a excepción de la región Norte que presenta la tasa más elevada, mientras se destacan las regiones Nordeste y Sul con la detección más baja. Todas las regiones elevan la detección a partir de la década del 70 , siendo la Sul la de detección más estable- 
mente baja. Las regiones Norte y Centro Oeste, mantienen las más altas detecciones de casos y los mayores incrementos, lo cual evidencia la desigual evolución regional de la endemia en el país ( Gráfico I).

Mientras, la observación de las diferencias al interior de las regiones según los estados que las integran, agudiza la expresión espacial diferenciada de la endemia.

Los estados que integran la región Norte, reconocida como de elevada intensidad de la endemia, presentan heterogénea e irregular detección de casos. En el territorio de Tocantins y Roraima elevados a la categoria de estados en 1998, la progresión de la endemia ocurre en las décadas del 70 y 80 respectivamente, cuando aún eran territorios. Los estados de Acre, Amazonas y Rondônia, mantienen elevada detección de casos hasta mediados de la década del 90 , en que son superados por los estados de Tocantins, Roraima e incluso el estado de Pará.

La región Nordeste, historicamente de baja prevalencia e incidencia en el país, presenta una evolución que distingue en especial a los estados de Maranhão y Piauí, com un incremento de la detección desde mediados de la década del 70, también apreciable com menor intensidad en los estados de Pernambuco y Ceará.

La región Centro-Oeste presenta la más crítica evolución de la endemia, en dos de sus estados: Mato Grosso y Goiás. Mientras, el estado de Mato Grosso do Sul, fragmentado de Mato Grosso en 1979, y el Distrito Federal elevado a esta categoria de território en la década de 60 , la detección se eleva a finales de la década del 70 , aunque distante de los estados mencionados anteriormente.

Por su parte, los estados de la región Sudeste se caracterizan por tasas relativamente estables, y el estado de Espírito Santo aparece com los mayores incrementos, seguido de Rio de Janeiro.

Por último, la región Sul del país, se destaca com la más baja incidencia y la evolución más estable en los estados de Santa Catarina y Rio Grande do Sul, mientras el estado de Paraná mantiene las más elevadas tasas de la región, durante todo el período estudiado.

\section{La evolución de la endemia en la segunda mitad del siglo XX. Control y expansión.}

El estudio de series históricas de tasa de detección en Brasil, para el periodo

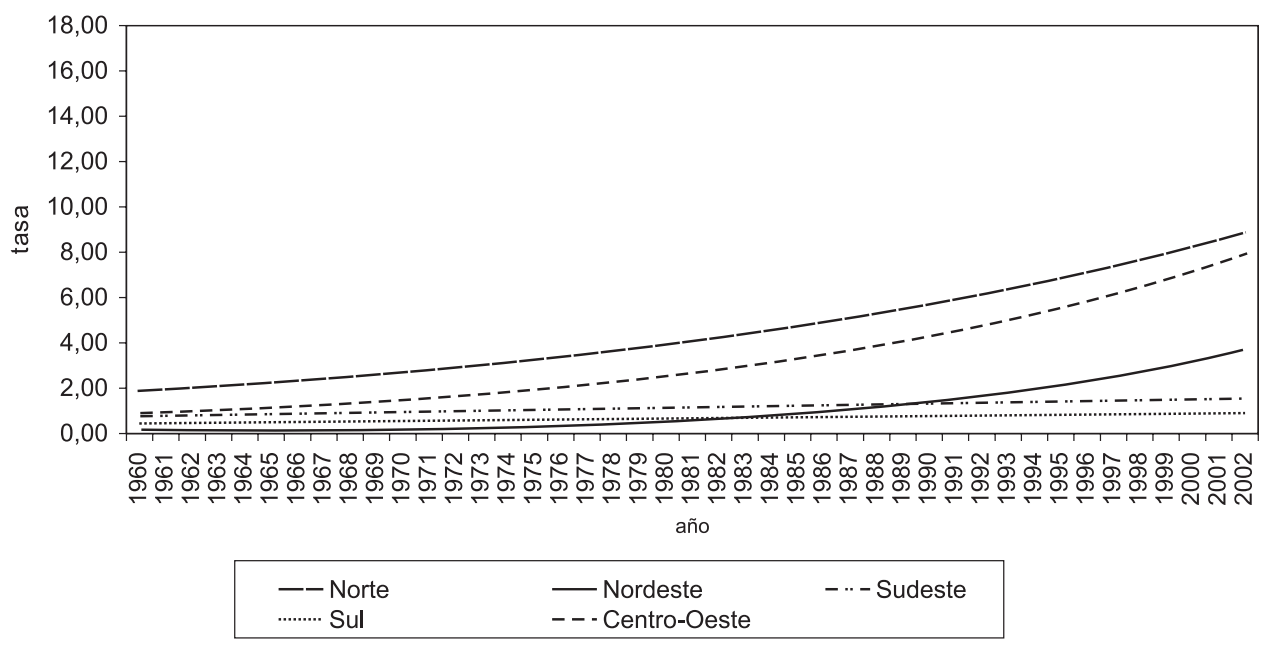

Fuente: ATDS.MINSA

Grafico 1 - Tasa de detección de lepra - Macroregiones - Brasil - 1960 - 2002

Chart 1 - Detection rates for Leprosy in Brazilian macro regions - 1960 -2002 
1950-1968, identifica una tendencia decreciente de la incidencia del orden del $3 \%$ al año ${ }^{10}$. Los mismos autores, estudiando el periodo de 1969 a 1987, observan una reversión de esta tendencia, con el incremento anual de casos del $6 \%{ }^{10}$.

Entre 1969 y 1985, un nuevo estudio de Zuñiga, concluye que la tendencia para el país era creciente, según este autor la situación sugería argumentos para fundamentar que el crecimiento era debido a factores de orden epidemiológico y no operacional ${ }^{11}$. Nuevos estudios realizados por el mismo autor en 1986 y 1987, concluyeron que la tendencia continuaba creciente y reiteraba que la base de esta era epidemiológica $^{12}$.

En 1989, Zuñiga observó un aumento de la endemia encima de lo esperado por el estudio de la tendencia, con un incremento de $34,8 \%$ de casos nuevos a nivel nacional, concluía este autor que la fase de estabilización de la endemia no estaba próxima y la endemia mostraba un peligroso potencial expansivo. Según la tendencia, las tasas de detección anual en el período de (1973 - 1988) eran ascendentes en todo el país, con un incremento medio de $5,38^{13}$.

En relación a las regiones, notaba el mayor ritmo de crecimiento en la Nordeste $(10,6 \%)$ y los menores en la Sul y Sudeste 3,74 y $3,38 \%$, respectivamente). Consideraba evidente la coexistencia de situaciones epidemiológicas diferentes al interior del país, y lo asociaba a la complejidad geográfica, climática, económica y social interna ${ }^{13}$.

La Coordinación Nacional de Dermatología Sanitaria, al analizar las tasas de detección anual para el periodo 1982 a 1992, concluyó que la tendencia era ascendente en el país, pero los estudios con que se contaba, no permitían afirmar si el crecimiento observado se debía a factores operacionales o epidemiológicos ${ }^{14}$.

Antes de la introducción de la PQT, la tasa de detección por formas clínicas, en el país, denotaba un incremento de las formas paucibacilares, asociado al incremento de la detección en edades más jóvenes, lo cual indicaba el real aumento de la transmisión y del nivel de la endemia ${ }^{11}$. Entre 1986 e 1993 se observó un incremento de 5,6 para 11,7/ 100.000 hab., de formas clínicas multibacilares; patrón no compatible con las características de alta endemicidad del país ${ }^{15}$. Según Martelli, ello pudiera ser explicado por una conducta conservadora en la cual, en caso de duda, se adoptaba un esquema de mayor duración (PQT/MB), y no un cambio real en el perfil epidemiológico del país ${ }^{15}$.

La afirmación en los trabajos de Zúñiga, de que el crecimiento de la incidencia era de orden epidemiológica, fue criticada por algunos especialistas, que consideraban que la reestructuración del programa de control, con el aumento de la cobertura, ampliación del diagnóstico, entrenamiento de personal de los servicios y campañas de divulgación de las señales y síntomas, había permitido, el hallazgo de muchos casos que antes no eran diagnosticados por deficiencias de los servicios de salud (prevalencia oculta). De esta forma estos especialistas, reforzaban la hipótesis de que eran factores operacionales los que influenciaban el crecimiento del número de casos.

Un nuevo estudio sobre la evaluación de la lepra en Brasil, para el período 1982 a $1994^{16}$, concluía que el indicador de detección en menores de 15 años, permitía distinguir los patrones epidemiológicos en el país y determinar el origen del crecimiento de la enfermedad, afirmando que el crecimiento del $43 \%$ observado en la detección de casos nuevos en este periodo, podía estar relacionado con la mejoría del sistema de información.

La autora citada, afirmaba que las áreas geográficas donde la lepra presentaba características endémicas o de expansión, según el indicador "tasa media de detección en menores de 15 años”, asociado al indicador "variación porcentual de la razón de las tasas medias de detección de 0 a 14/15 y más años", en los años de 1990 a 1994, comprendían los estados de: Per- 
nambuco, Espírito Santo, Maranhão, Piauí, Mato Grosso, Acre, Pará, Rio de Janeiro, Paraíba, Alagoas, Bahia y Sergipe ${ }^{16}$.

El análisis de la serie histórica de la tasa de detección en el estudio de Andrade, de 1996, sugirió que la interpretación del perfil de expansión de la endemia debería ser revisado, considerando la importancia de factores operacionales, a partir de la década del 80. Concluía así que el crecimiento de la tasa de detección no corresponde necesariamente a un incremento de la probabilidad de enfermar ${ }^{16}$.

En el periodo 1982 - 1995, el análisis de los indicadores de proporción de casos en menores de 15 años, la distribución por formas clínicas y el porcentaje de casos nuevos detectados con deformidad, sugería que la endemia estaban en expansión y que mantenía un comportamiento diferenciado entre Unidades Federadas ${ }^{1}$.

El estudio sobre el comportamiento de la lepra en Brasil ${ }^{17}$, en la década de 1986 a 1996, concluyó que, a excepción del estado de Rio Grande do Sul, la lepra continuaba como un problema de salud pública en el país, según criterios de la OMS. Se afirmaba también que aún era elevada la prevalencia oculta, lo cual dificultaría el cumplimento de la meta de eliminación como problema de salud publica en el año 2000.

Según estudios de Andrade 1996, en la década del 60 y hasta mediados de los 70, la tasa de prevalencia en Brasil permanece prácticamente constante. A partir de1976 asciende hasta alcanzar un pico en 1990 , con una prevalencia de 18,5 por 10 000 hab. Comienza a partir de este año un nuevo descenso ${ }^{18}$.

El incremento de la prevalencia en los 80 , se ha asociado a factores como la descentralización de las acciones, el fortalecimiento del programa de control y la aplicación de la terapéutica en gran escala (PQT/OMS), iniciada en 1986, o hasta procedimientos aplicados en relación con las altas o los enfermos fuera de control. De esta forma podría no indicar un incremento del nivel de endemicidad en el país ${ }^{19}$.

Según la OMS, la disminución de la prevalencia oculta es la principal causa de crecimiento del número de casos en los países endémicos, incluyendo Brasil, una vez que cuando se mejora la calidad y se incrementan los servicios, disminuye la demanda reprimida por mejoramiento del acceso de la población al diagnóstico.

No obstante el mantenido descenso de la prevalencia a partir de 1991 coincide con la introducción, por el Ministerio de la Salud, del esquema oficial de la poliquimioterapia, con tratamiento de dosis fija (6 dosis para las formas pauci y 24 para las multibacilares), revelando la importancia de este factor operacional en tal reducción.

La reducción de la prevalencia en Brasil, ocurrió en menores niveles que los presentados en los demás países endémicos. Este hecho probablemente se relaciona con la adopción más tardía de la poliquimioterapia, además de peculiaridades administrativas del programa como el mantenimiento en el registro activo de pacientes en abandono, por 2 años para los paucibacilares y 4 años para los multibacilares. En otros países, se adoptó el criterio de la OMS, donde los pacientes que no comparecían al servicio en el periodo de 12 meses (abandono), eran retirados del registro activo. Según Martelli et al, 2002, la prevalencia es reflejo de la PQT y no un declino natural de la endemia ${ }^{20}$.

\section{Evolución del poblamiento y diferenciación geográfica de la endemia de la lepra}

Es particularmente importante reafirmar la no relación entre las regiones más pobladas y las de mayor detección de casos de lepra, aunque en todas las regiones el crecimiento poblacional se localizó fundamentalmente en áreas urbanas.. Mientras en la década del 40, el grado de urbanización del país era de $30 \%$ aproximadamente, en el 2000, el $85 \%$ de la población residía en espacios urbanos ${ }^{21}$.

Los movimientos migratorios se consideran decisivos en este incremento, con más débil participación de las diferencias 
rural-urbana de las tasas de mortalidad y fecundidad, dado que como se conoce el crecimiento vegetativo ha sido tradicionalmente más bajo en áreas urbanas, en especial en las de mayor tamaño poblacional.

La región Centro-Oeste es la de cambios más significativos, en 1940 era la menos urbanizada con apenas el $20 \%$ de la población en espacios urbanos y en la actualidad aproximadamente esta proporción se encuentra en áreas rurales. La región Norte alcanzó la mayor proporción del crecimiento en áreas rurales, mientras la región Nordeste solo a partir de la década del 70 la población de esta región muestra una reducción absoluta de su población rural, que puede interpretarse tanto por la migración ruralurbana, como por la caída de fecundidad ${ }^{21}$.

Cabe señalar que a pesar de la disminución de las tasas de migración urbana, esta población se incrementó en la década del 90 en más de 3 millones de personas. Otra importante observación es que la atracción de la región Norte que a partir de la década del 70 recebió grandes contingentes de inmigrantes que se localizaron tanto en áreas rurales como urbanas, pierde población rural durante la década del 90, como expresión de la falta de éxito de los programas de ocupación, asociados entre otros factores a la incidencia de enfermedades, en especial la malaria.

Pérdida de población rural y concentración de la población en ciudades mar- ca el proceso de diferenciación espacial de la población brasileña desde la mitad del siglo pasado. Las diferencias espacio-temporales están determinados por las peculiaridades de las transformaciones de la organización espacial, en especial de la dinámica entre el éxito y estancamiento de los procesos de ocupación. Mientras, el crecimiento vegetativo se mantuvo en general más elevado en las áreas de emigración ( Gráfico II).

Explorando otros indicadores de la movilidad de la población en la primera mitad de la década del 90, podemos identificar la reiteración de las anteriores consideraciones. Uno de los indicadores que permiten analizar la migración inter e intraregional es la referente a la naturalidad de la población en relación al municipio y Unidad de la Federación de residencia actual.

Según las conocidas Investigaciones Nacionales por muestra de domicilio del IBGE, en 1999, último año disponible, se observa que en las regiones Norte y Centro-Oeste, se concentran los mayores porcentajes de personas no naturales de la Unidad de la Federación, mientras las proporciones de población no naturales del municipio de residencia actual presenta valores similares, alejándose la región Nordeste con la menor y la Centro-Oeste, que supera el $50 \%$ del total.

Las anteriores consideraciones, a pesar de su generalidad, indican una asocia-

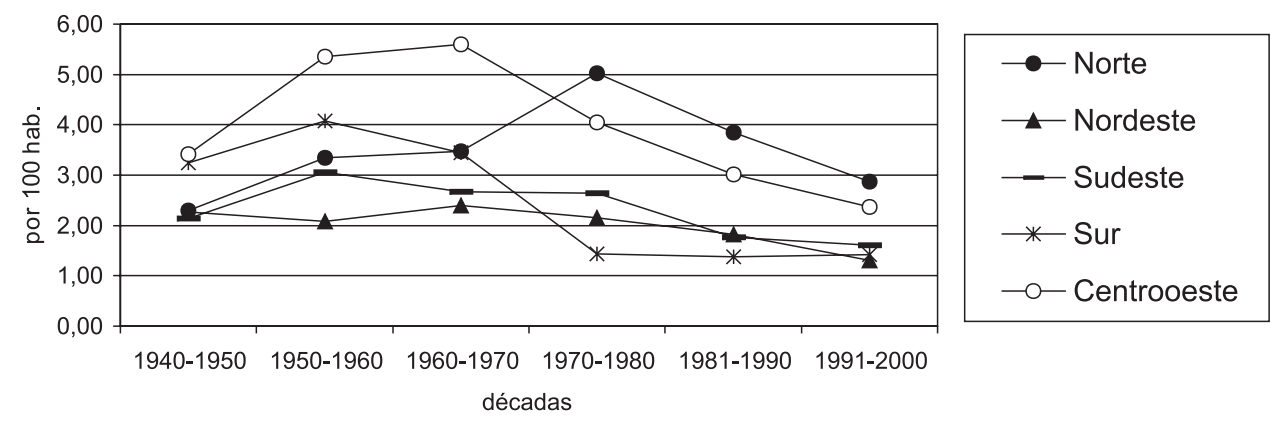

Fuente:IBGE - Estatísticas do século XX

Grafico 2 - Tasa geométrica de crecimiento poblacional medio annual por regiones - Brasil $1940-2000$

Chart 2 - Geometric rates of mean annual population growth in Brazilian macro regions - 1940 2000 
ción de la diferenciación intraregional e interregional de la endemia y el crecimiento de la población. Según el censo de 1996, las mayores proporciones de personas no residentes en 1991, se encuentran en los estados de Tocantins (8.08\%) y Rondônia (5.28\%). Los tres estados de la región Centro-Oeste también presentan elevados porcentajes de población no residente: Mato Grosso do Sul, 7.66\%; Mato Grosso, $6.72 \%$ y Goiás, $6.39 \%$. Por su parte, el Distrito Federal alcanza el mayor valor entre las Unidades de la Federación con 9.16\% de la población no residente. En el resto de los estados las proporciones son alrededor del $2 \%$ e inferiores.

Las regularidades sobre la evolución espacio-temporal de la incidencia de la lepra, y las observaciones realizadas sobre la evolución del crecimiento de la población regional y estatal, muestran grandes coincidencias. Los estados de más alta detección y con evolución más desfavorable de la endemia, presentaron altos incrementos de población, en especial en las décadas del 60 y 70.

Cabe enfatizar que las regiones Norte y Centro-Oeste, que obtuvieron las más altas tasas de crecimiento poblacional, registraron a mitad de la década del 90, los mayores porcentajes de población migrante, y el más intenso crecimiento de la tasa de detección de casos de lepra. Por su parte los estados que evidencian una tendencia creciente de sus tasas de detección, también coinciden con los de mayor proporción de población no natural de estos territorios y provenientes de estados de alta detección de casos.

\section{Conclusiones}

La reconstrucción de la historia reciente de la diferenciación territorial de la endemia de la lepra, según regiones y estados en Brasil, permite distinguir una desigual evolución espacio-temporal interregional, intraregional e interestatal, como las que adelante serán descritas.

La distribución actual de la lepra en Brasil, demuestra su concentración en 14 de los 27 estados del país. En los siete de la región Norte (Rondônia, Acre, Amazonas, Roraima, Pará, Amapá e Tocantins); cuatro de la región Nordeste (Maranhão, Piauí, Ceará y Pernambuco); dos de la región Centro-Oeste (Mato Grosso y Goiás) y uno de la región Sudeste (Espírito Santo). En estos estados se concentra aproximadamente el $80 \%$ de las notificaciones del periodo estudiado.

Los estados de más alta detección y con evolución más desfavorable de la endemia, presentaron elevados crecimientos de la población, en especial en las décadas del 60 y 70. Las regiones Norte y Centro Oeste, obtuvieron las más altas tasas de crecimiento poblacional. Registraron a mitad de la década del 90, los mayores porcentajes de población migrante, y el más intenso crecimiento de la tasa de detección de casos de lepra.

La permanencia histórica de elevada incidencia de la lepra en la región Norte, en el estado de Maranhão y Pernambuco en la región Nordeste, del estado de Paraná en la región Sul, así como relativamente bajas incidencias en el resto de esta última región, sugieren la existencia de contextos geográficos de diferente vulnerabilidad a la producción social de la lepra, y de nuevos componentes en la organización espacial en algunos territorios. Aunque aceptamos la posible influencia de factores operacionales en la construcción de estas regularidades, es poco probable que estos se hayan mantenido durante más de 70 años. 


\section{Referências}

1. Ministério da Saúde. Relatório de avaliação do programa de controle da hanseníase. Coordenação Nacional de Dermatologia Sanitária, Fundação Nacional de Saúde. Ministério da Saúde, 1997, mimeo.

2. Valverde B. Prophylaxia da Lepra. Rio de Janeiro: 1915, passim.

3. Magalhães JL. Lepre au Brasil. Rio de Janeiro: Typ. Pereira Braga, 1900.

4. Araújo Souza HC. História da lepra no Brasil. Rio de Janeiro: Departamento de Imprensa Nacional, Vol. II y III, 1956, passim.

5. Rodríguez M. Freqüência da lepra no Estado de Maranhão. VII Congresso Brasileiro de Medicina. Rio de Janeiro, 1918, passim.

6. Prado Júnior C. Formação do Brasil Contemporâneo. São Paulo: Brasiliense, 1987, passim.

7. Lechat MF., Vanderveken, M. Indicadores Epidemiológicos Básicos para la Vigilancia de la Lucha contra la Lepra. Washington, DC. POS, 1984, passim.

8. Irgens L. Secular Trends in Leprosy: Increase in Age at Onset Associated with Declining Rates and Long Incubation Periods. Int J Leprosy 1985:610-7.

9. Magalhães MCC. Avaliando a cobertura da vacina BCG en contatos de hanseníase. Trabalho apresentado no $16^{\circ}$ Congresso Internacional de Hanseníase. Salvador, Bahia, 2002, mimeo.

10. Motta CP, Zuñiga MG. Time Trends of Hansen's Disease in Brazil. Int J Leprosy 1990: 453-61.

11. Zuñiga MG. Informe de Assessoria al Programa de Control de la Enfermidad de hansen en Brasil. Coordenação Nacional de Dermatologia Sanitária, Ministério da Saúde, 1987, mimeo.

12. Zuñiga MG. Informe de Assessoria al Programa de Control de la Enfermidad de hansen en Brasil. Coordenação Nacional de Dermatologia Sanitária, Ministério da Saúde, 1988, mimeo.
13. Zuñiga MG. Informe de Assessoria al Programa de Control de la Enfermidad de hansen en Brasil. Coordenação Nacional de Dermatologia Sanitária, Ministério da Saúde, 1989, mimeo.

14. Ministério da Saúde. Fundação Nacional de Saúde. Centro Nacional de Epidemiologia. Coordenação Nacional de Dermatologia Sanitária. Estudos de Tendência de Hanseníase, 1992, mimeo.

15. Martelli CMT et al. Changes in leprosy clinical pattern after multidrug therapy implementation. Int J Leprosy 1995: 95-7.

16. Andrade VLG. Evolução da hanseníase no Brasile perspectivas para a sua eliminação domo problema de saúde pública. Tese de doutorado. Rio de Janeiro: Escola Nacional de Saúde Pública/Fundação Oswaldo Cruz, 1996, passim.

17. Pereira GFM. Características da hanseníase no Brasil. Situação e tendência no perído de 1985 a 1996. Dissertação de mestrado. São Paulo: Escola Paulista de Medicina, 1999, passim.

18. Andrade V, Sabroza PCT, Albuquerque MFM. Séries temporais dos indicadores de morbidade da hanseníase Brasil, 1946-1994. IESUS 1996; V(3): 23-41.

19. Ministério da Saúde. Fundação Nacional de Saúde. Centro Nacional de Epidemiologia. Coordenação Nacional de Dermatologia Sanitária. Hansen's disease in Brazil progress towards elimination. 1998, mimeo.

20. Martelli CMT, Stefani MMA, Penna GO, Andrade ALSS Endemias e epidemias brasileiras, desafios e perspectivas de investigação científica: hanseníase. Rev Bras Epidemiol 2002: 273-85.

21. IBGE. Taxa geométrica de crescimento da população residente, segundo as grandes Regiões e Unidades da Federação. In: Estatísticas do Século XX, 1940-2000, passim.

Recebido em: 16/06/05

Versão reformulada reapresentada em: 13/09/05

Aprovado em: 28/09/05 